\title{
Percepciones de docentes de ingeniería de México sobre componentes personales, profesionales e institucionales que intervienen en su formación
}

\author{
Engineering Professors' Perceptions on Personal, Professional, and Institutional \\ Components Involved in Their Training
Percepções de docentes de engenharia do México sobre componentes pessoais, profissionais e institucionais envolvidos na sua formação

Fernando Banda-Muñoz

Universidad Autónoma de Nuevo León Facultad de Ingeniería Mecánica y Eléctrica

Nuevo León, México

fernando.bandam@uanl.mx

https://orcid.org/0000-0002-0155-9696

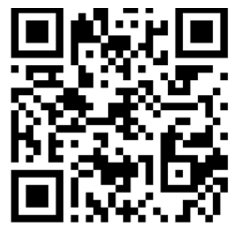

Arnulfo Treviño-Cubero

Universidad Autónoma de Nuevo León Facultad de Ingeniería Mecánica y Eléctrica

Nuevo León, México

arnulfo.trevinoc@uanl.mx

https://orcid.org/0000-0002-0958-8352

Aldo Raudel Martínez-Moreno Universidad Autónoma de Nuevo León Facultad de Ingeniería Mecánica y Eléctrica

Nuevo León, México

aldo.martinezm@uanl.mx

https://orcid.org/0000-0001-9550-2182

Recibido • Received • Recebido: 15 / $11 / 2019$

Corregido • Revised • Revisado: 20 / 07 / 2021

Aceptado•Accepted • Aprovado: 23 / 08 / 2021

\begin{abstract}
Resumen: Este artículo trata sobre la educación en ingeniería, enfocado al proceso de formación del profesorado, en carreras de ingeniería de la Universidad Autónoma de Nuevo León. El objetivo consiste en analizar factores personales, institucionales y del proceso de formación que se relacionan con la formación del profesorado de ingeniería. La metodología utilizó como instrumento una encuesta que se aplicó a 51 docentes de diez carreras de ingeniería, que se seleccionaron por muestreo aleatorio simple. Para recolectar información se aplicaron escalas de Likert, preguntas de selección y preguntas abiertas. Se aplicó una técnica de visualización para la representación de conceptos recurrentes. El enfoque de la investigación es descriptivo y se orientó a explicar particularidades sobre la formación de profesorado en el contexto específico. Se definieron variables, por ejemplo, la satisfacción con actividades de
\end{abstract}


http://doi.org/10.15359/ree.25-3.32

http://www.una.ac.cr/educare

educare@una.ac.cr

superación, o los temas que proponen incluir en dichas actividades, las cualidades ideales del personal docente de ingeniería, y otras que tienen repercusión en la formación para el desempeño docente. Los resultados aportaron criterios para identificar áreas de formación del personal docente, y conformar actividades con la finalidad de favorecer el desarrollo del profesorado de ingeniería. Se puede concluir que es adecuada la satisfacción del profesorado con la formación que ha alcanzado, y los resultados aportaron una base para continuar la investigación con alcance transformador, mediante propuestas específicas que propicien la formación de docentes de acuerdo con sus necesidades personales, profesionales y que se correspondan con las exigencias de la sociedad actual.

Palabras claves: Proceso formativo; educación en ingeniería; formación del profesorado; perfil del profesorado de ingeniería.

Abstract: This article is about education in engineering, focused on faculty members' training process in engineering majors at the Universidad Autónoma de Nuevo León. The objective relies on analyzing personal and institutional factors and the training process related to the engineering faculty training. The methodology used a survey as an instrument. The survey was done on 51 professors from ten engineering majors; the professors were selected by simple random sampling. Likert scales, multiple-choice questions, and open questions were applied to collect data. A visualization technique was implemented to represent the recurring concepts. The approach of this research was descriptive and oriented to explain particularities about the training of faculty members in a specific context. Variables were determined, such as the satisfaction of achievement activities, the topics proposed to be included in the activities mentioned above, the ideal qualities of engineering faculty members, and other variables that have repercussions in training for faculty performance. The results contributed criteria to identify the areas of training of the faculty and constitute activities to encourage engineering faculty development. It can be concluded that the satisfaction of the faculty is adequate with the achieved development. The results provided a basis for continuing research with a transformative scope, through specific proposals to enhance faculty training according to their personal and professional needs and in agreement with society's current demand.

Keywords: Training process; engineering education; teacher training; engineering teacher profile.

Resumo: Este artigo sobre a educação em engenharia, está enfocado no processo de formação de docentes nas carreiras de engenharia, na Universidade Autônoma de Nuevo León. O objetivo é analisar fatores pessoais, institucionais e de processo de treinamento relacionados à formação do docente de engenharia. A metodologia utilizou como recurso uma pesquisa aplicada a 51 professores de dez carreiras de engenharia, selecionados por amostragem aleatória simples. Escalas Likert, questões de seleção e questões abertas foram aplicadas para coletar informações. Uma técnica de visualização foi aplicada para a representação de conceitos recorrentes. A abordagem da pesquisa é descritiva e focada na descrição de particularidades sobre a formação de professores no contexto específico. As variáveis foram definidas, por exemplo, satisfação com as atividades de melhoria ou os tópicos que eles propõem incluir nessas atividades, as qualidades ideais de um professor de engenharia e outros que têm impacto no treinamento para o desempenho do professor. Os resultados forneceram critérios para identificar áreas de formação de professores e modelar atividades para favorecer o desenvolvimento de professores de engenharia. Conclui-se que a satisfação dos professores com a capacitação realizada é adequada, e os resultados forneceram base para a continuidade de pesquisas 
http://doi.org/10.15359/ree.25-3.32

com alcance transformador, por meio de propostas específicas que promovam a capacitação dos professores de acordo com suas necessidades pessoais, profissionais e que correspondem às demandas da sociedade atual.

Palavras-chave: Processo de formação; formação em engenharia; formação de professores; perfil de professores de engenharia.

\section{Introducción}

La educación superior enfrenta el reto de preparar a docentes del siglo XXI, pues son actores clave del proceso de formación profesional. En las carreras de ingeniería se puede considerar un perfil general de la persona docente, caracterizado por aspectos comunes tales como las competencias transversales, que se complementan con otras competencias específicas según particularidades de las respectivas carreras.

Este trabajo expone resultados de la labor investigativa de los autores en relación con la formación del profesorado de ingeniería, y se nutre de las vivencias profesionales en el ejercicio de sus funciones. En tal sentido, las preocupaciones investigativas se relacionan con la necesidad de profundizar en el conocimiento del proceso de formación del profesorado universitario de ingeniería.

El enfoque de esta investigación se orientó a describir particularidades de un conjunto de aspectos que tienen relevancia en la formación del profesorado de ingeniería, en el contexto específico en que se investigó. Se tiene como premisa que la formación de la persona docente de ingeniería es un fenómeno que tiene múltiples formas de análisis, y todas ellas contribuyen a orientar las acciones de diversos actores para elevar la calidad de su preparación, desde las actividades que se promueven a nivel institucional hasta la autogestión de la formación profesoral.

Es conocido que el profesor y la profesora de ingeniería en México, al igual que en otros países, no poseen formación para la labor docente; significa que su preparación se logra casi de manera espontánea, ya sea a través de cursos de capacitación, de actualización o de manera autodidacta. Por eso constituye un problema de investigación permanente la exploración de los componentes esenciales que influyen en la satisfacción de necesidades de superación docente. Es común que en la enseñanza de ingeniería, como apunta Cederbaum (2018), se practique con fórmulas aprendidas para después usar ese mismo conocimiento aplicando fórmulas aprendidas, lo que se traduce en un procedimiento mecánico. Si bien es cierto que en esta disciplina el método científico es imprescindible en la formación del ingeniero y la ingeniera, también se necesita desarrollar procesos lógicos de pensamiento y habilidades personales y sociales que solo pueden ser desarrolladas a partir de una persona docente con una sólida formación.

De acuerdo a lo expresado, la búsqueda de formas y vías para lograr una formación más efectiva del personal docente de ingeniería continúa en el centro de la atención de las investigaciones educativas, así como de los organismos y organizaciones cuyo objeto lo constituye la persona especialista en esta rama. 
http://doi.org/10.15359/ree.25-3.32

http://www.una.ac.cr/educare

educare@una.ac.cr

En las condiciones específicas del estudio realizado, su objetivo corresponde al análisis de factores relacionados con la formación del profesorado de ingeniería; personales, institucionales y del proceso de formación profesional de la persona estudiante, y fueron precisados en variables de tipo cualitativo y descriptivo. Es por ello que sus resultados se expresan en las percepciones sobre aspectos tales como la satisfacción con la superación que ha recibido el personal docente, las cualidades deseadas en el profesorado de ingeniería, y las temáticas que son de interés para capacitarse en función de su desempeño, entre otros aspectos.

\section{Marco teórico}

\section{La formación docente para universidad y el perfil del profesorado de ingeniería}

El profesorado universitario requiere desarrollar cualidades de idoneidad ética, científica y pedagógica, y esto da lugar a que las características personales y profesionales que lo distinguen sean objeto de investigaciones (Álvarez 2011). De ahí que se definen criterios; por ejemplo, que demuestre "deseos de crecimiento, con altos estándares de calidad, seguro de su desempeño frente a sus pares académicos, con títulos de maestría y doctorado, con suficiencia en el manejo de una segunda lengua que le permita interactuar con sus pares académicos a nivel internacional y realizar investigaciones que aporten al conocimiento de su disciplina académica" (de Castro 2011, pp. 33-34, citando a Mestre, 2007).

En México existen programas derivados de políticas institucionales y se organizan actividades hasta los niveles de facultad y carrera. A nivel nacional se pueden citar ejemplos como el Programa Nacional de Formación de Profesores, desarrollado por la Asociación Nacional de Universidades e Instituciones de Educación Superior; el Programa Nacional de Superación del Personal Académico, y el Programa de Mejoramiento del Profesorado (Zaldívar Acosta et al., 2018).

Esta investigación está en concordancia con la tendencia actual de las universidades de enfocarse a una formación más integral de sus egresados y egresadas, y por lógica esto abarca también la formación docente en cualidades personales, así como en un fortalecimiento de sus competencias (Castillo-Elizondo et al., 2019).

Sin embargo, los ingenieros e ingenieras con vocación docente generalmente no poseen una sólida formación pedagógica (Cáceres Mesa et al. 2003), lo que justifica las actividades de preparación para el desempeño de sus funciones. Esto resulta más relevante cuando el profesorado no posee mucha experiencia; según Fondón et al. (2010), entonces se debe superar en ámbitos de la enseñanza, de las relaciones interpersonales y de la gestión o el contexto institucional. Por otra parte, las personas empleadoras plantean a la universidad determinadas exigencias sobre las personas egresadas de ingeniería, básicamente en competencias y cualidades del perfil de egreso (Centro de Investigación para el Desarrollo [CIDAC], 2014; VegaGonzález, 2013). Dichas consideraciones repercuten en la preparación del personal docente para que sea capaz de impactar en la formación de sus estudiantes, lo que significa que debe perfeccionar su docencia para ponerse a la altura de las demandas de las personas empleadoras. 
http://doi.org/10.15359/ree.25-3.32

Deacuerdo con Duque et al.(2011), quien enseña ingeniería requiere poseer conocimientos de la disciplina que imparte, de sus orígenes y de su relación con otras disciplinas, contar con formación pedagógica para ejercer la enseñanza de dicha materia, tomar en cuenta la elaboración de proyectos con beneficios sociales, y dominar el perfil de la persona estudiante que forma. En la actualidad el perfil docente de ingeniería ha de incorporar las exigencias actuales de las personas empleadoras, que cada día marcan características más integrales y complejas a este tipo de profesional.

En correspondencia con lo anterior, y según las posiciones teóricas, cobran interés otras dimensiones del perfil docente en ingeniería para su mejor desempeño en el proceso de formación profesional, por ejemplo: la orientación del aprendizaje basado en la investigación, la organización del aprendizaje en situaciones prácticas de la carrera, y el fomento de la innovación y el liderazgo en el propio proceso formativo. Pero además, el profesor y la profesora de ingeniería son parte de una comunidad académica y científica y conviven con actores diversos; de ahí que requieren manifestar un comportamiento humano (Vega-González, 2013). En esa misma dirección se afirma que necesitan relacionarse positivamente y cooperar con los demás (Cid Reborido, 2009). Esto último se vincula con las competencias para el trabajo en equipo, que es muy importante para la educación en ingeniería (Beddoes y Panther, 2018).

Los criterios expresados coinciden con las tendencias actuales en la formación de ingenieros e ingenieras para el siglo XXI, que apuntan al desarrollo de modelos que tengan en cuenta"la relevancia social y el aprendizaje de la ingeniería de cara al entorno real de la profesión" (Banda, 2018, p. 79). Además, si se toma en consideración la complejidad de las carreras de ingeniería, entonces en el perfil del profesorado se debe desarrollar una competencia para integrar saberes; estos se ubican en las ciencias técnicas, ciencias de la educación, y otros temas transversales que le resultan indispensables.

Desde el punto de vista del desarrollo institucional, se enfoca el fortalecimiento del trabajo del personal docente, con énfasis en componentes pedagógicos, didácticos, éticos e investigativos. Para ello se realizan actividades que se fundamentan según variados términos, tales como superación, preparación, capacitación del personal docente (Salinas Cruz, 2019), o desarrollo profesional de dicho personal (López Serrano, 2019).

Se enfatiza en incorporar la dimensión de desarrollo humano; es decir, la formación no debe concebirse como un proceso estrictamente técnico (de Castro, 2011). Pero lo esencial es que ocurra un proceso formativo, mediante el cual la persona docente logre un desarrollo de sus cualidades personales, profesionales y sociales que le permitan un mejor desempeño. La formación docente se lleva a cabo por variadas modalidades, incluyendo la propia autogestión de su superación y llegando a formas más complejas, como la obtención de titulaciones de maestría y doctorado. 
http://doi.org/10.15359/ree.25-3.32

http://www.una.ac.cr/educare

educare@una.ac.cr

\section{La formación del profesorado de ingeniería para la innovación y el liderazgo}

Entre las problemáticas más actuales y relevantes relacionadas con el rol profesoral en ingeniería se encuentran el fomento de la innovación y el liderazgo. Con respecto a esto, una encuesta nacional a las personas empleadoras en México (CIDAC, 2014) reveló que las competencias más escasas en quienes egresaron fueron cultura general (10.69\%), liderazgo (9.65\%), innovación (8.23\%) y eficiencia personal (8.23\%). Los estudios realizados sobre esto critican las metodologías que limitan al estudiantado al papel de receptor de información y se resalta la importancia de la innovación, la creatividad y el diseño en quien imparte ingeniería (Vega-González, 2013). Se coincide con Pusca y Northwood (2018) sobre la atención que debe prestar el docente o la docente a las actividades de aprendizaje donde se estimulen la curiosidad y la investigación en su clase.

Para el profesorado la innovación se expresa también en el mejoramiento de su propia práctica docente (Más Torelló, 2011); esto se concreta cuando elabora material didáctico, establece relaciones con el entorno socioproductivo, participa en la mejora del proceso formativo, evalúa su calidad e impacto, y autogestiona su propia superación. Relacionado con lo anterior, la elaboración y gestión de proyectos de investigación de carácter educativo, de desarrollo institucional, o de investigación sobre objetos de la ingeniería, constituyen una fuente de formación para el personal docente.

Un concepto que destaca es el liderazgo; en principio se enfoca en las personas directivas (Vázquez Toledo et al., 2015), pero ocurre también en otros actores de la universidad. En tal sentido, el personal docente de ingeniería debe incidir en el estudiantado para que las personas egresadas cuenten con habilidades de liderazgo (Flores Aguilar et al., 2018). De aquí se infiere que la formación del liderazgo en el estudiantado presupone que el profesorado lo pone en práctica también. Resultaría contradictorio pensar en competencias de liderazgo en el estudiantado si el profesorado no actúa para propiciarlo, y ello requiere preparar a la persona docente para incorporarlo al proceso de formación.

De lo planteado por Albor Calderón et al. (2018), se pueden precisar componentes de liderazgo en el profesorado de ingeniería, que tendrían repercusión en sus estudiantes: toma de decisiones, autoconfianza, sentido de responsabilidad, iniciativa, y capacidad de motivar a las demás personas. Además, se aprecia que los conceptos de liderazgo y emprendimiento aparecen muy mencionados de conjunto en la formación en ingeniería (Teerijoki y Murdock, 2014).

En su proceso de desarrollo profesional el personal docente participa en actividades que se organizan por diferentes instancias de la institución; estas se realizan a través de estrategias, programas de formación, proyectos específicos y otras vías. También se tiene en cuenta el proyecto de vida profesional autogestionado. La preparación del profesorado es una base para el impacto que pueda lograr en la formación de sus estudiantes. 
http://doi.org/10.15359/ree.25-3.32

\section{Metodología}

Esta investigación situó su interés en el profesorado de ingeniería en la Universidad Autónoma de Nuevo León, México; pero se debe precisar que no se centró en características asociadas directamente con cada uno de los perfiles específicos por carreras. La perspectiva se orienta hacia elementos comunes a las carreras de ingeniería, tales como cualidades del profesorado, y contenidos de capacitación adecuados para ejercer el trabajo docente, entre otros.

El presente estudio constituye una investigación no experimental con una aproximación al tipo mixto y con un diseño metodológico descriptivo de corte transversal que es conveniente para el enfoque descriptivo; de manera que no se interviene en la realidad estudiada, y solo se observa el objeto o fenómeno en su situación real, se describe y se realizan valoraciones (Denzin y Lincoln, 2012; García Salinero, 2004). Se utilizó la estadística descriptiva básica para realizar cálculos numéricos que conduzcan a obtener datos esenciales, tales como promedios y frecuencias.

Se aplicó una encuesta a través de Google Forms a profesorado de ingeniería de las carreras Administrador de Sistemas, Aeronáutica, Electrónica y Automatización, Electrónica y Comunicaciones, Manufactura, Ingeniero en Materiales, Mecánico Administrador, Mecánico Electricista, Mecatrónica, y Tecnología de Software. Con anterioridad fue sometida a un pilotaje que permitió corregir la redacción de algunos de los ítems. A las personas participantes se les informó del objetivo de esta y además se les aseguró el anonimato de sus respuestas.

La selección de participantes se hizo por muestreo aleatorio simple, abarcando representación de las carreras mencionadas. Se aplicó un cuestionario a 51 profesores y profesoras. La composición por sexo fue de 27 hombres y 24 mujeres; poseen título de Doctor y Doctora el $21.5 \%$, y de Maestro o Maestra el 60.7\%; el 49.0\% tiene más de 10 años de experiencia docente, y se puede considerar que el $51.0 \%$ está en un proceso de desarrollo de sus potencialidades como docentes.

Se aplicaron escalas de Likert; con este procedimiento se obtiene la opinión de un sujeto en base a su grado de acuerdo o desacuerdo. Cada enunciado utilizó cinco categorías y con el total de respuestas se calculó el valor medio de cada aspecto sometido a consideración, que se puede comparar con la escala graduada de 5 puntos como máximo. En base a estas preguntas se realizó también análisis de frecuencias. Se utilizaron preguntas de selección múltiple según un listado. Se aplicó la técnica de visualización, que consiste en la representación gráfica de las palabras más recurrentes, de modo que los diferentes tamaños expresan la importancia de cada concepto que forma la nube de palabras (Peña Pascual, 2012). Se obtuvo información mediante preguntas abiertas, de dos tipos; en unas se limitó la cantidad de enunciados a responder, y debían expresarse en una palabra; en otros casos se podían aportar enunciados libremente y con más de una palabra. Para el análisis de los resultados se determinaron categorías de acuerdo las respuestas dadas para una mejor comprensión de los resultados obtenidos.

Las categorías que se investigan mediante la encuesta se agruparon en tres ámbitos principales que se relacionan a continuación. En cada uno de ellos se identificaron indicadores 
http://doi.org/10.15359/ree.25-3.32

http://www.una.ac.cr/educare

educare@una.ac.cr

obtenidos de la revisión de la literatura y que a su vez se utilizaron para la elaboración de los ítems de la encuesta.

a) Percepción del profesorado respecto a la institución y el proceso de formación del personal docente:

- Percepción de la persona docente sobre las instancias institucionales que aportan a su formación.

- Propuestas del personal docente sobre tipos de actividades que prefieren, e intereses en temáticas que son necesarias para la preparación del personal docente.

b) Sobre la práctica docente en el proceso formativo:

- Percepción del grado de protagonismo del estudiantado en su aprendizaje

- Criterios sobre actividades docentes que están presentes en la formación de su alumnado

- Valoración sobre temas pertinentes y sobre prioridades a considerar por la persona docente en la formación ingenieril

- Competencias transversales que se deben fortalecer en el proceso formativo de la persona estudiante.

\section{c) Ámbito personal y profesional del personal docente:}

- Satisfacción del personal docente con su formación

- Percepción de cambios por parte del personal docente

- Cualidades relacionadas con el perfil del personal docente

\section{Resultados y discusión}

A continuación, se analizan los resultados obtenidos en cada uno de los tres ámbitos mencionados con anterioridad a partir de los indicadores señalados. Este análisis incluyó los procedimientos metodológicos de tipo cualitativo y cuantitativo.

a) Percepción del profesorado respecto a la institución y el proceso de formación del personal docente

Percepción de la persona docente sobre las instancias de la institución que aportan a su formación

El profesorado valora que las actividades de mayor aporte son las que se organizan a nivel de universidad, que obtiene promedio de 4.41 en escala de 5 puntos; en segundo lugar, ubican 
http://doi.org/10.15359/ree.25-3.32 http://www.una.ac.cr/educare educare@una.ac.cr

a la Coordinación Académica de la carrera, seguido de la preparación de modo autodidacta, la Subdirección Académica y el Departamento de Capacitación respectivamente.

En tal sentido, se observó que existen importantes vías de desarrollo del personal docdente, que son las relaciones con otras instituciones nacionales o internacionales, y estas se ubicaron en los últimos lugares con 3.35 y 3.62 de promedio. Si se tiene en cuenta que son modalidades para lograr mayor nivel del profesorado, se debe enfatizar en las relaciones interinstitucionales y la movilidad. La gestión formativa del profesorado por estas vías permite acceder a becas, estancias de investigación, programas de maestría o doctorado, proyectos de investigación y estudios en instituciones de avanzada (Figura 1).

Figura 1: Percepción del personal docente sobre diferentes instancias que aportan a su formación. Promedios

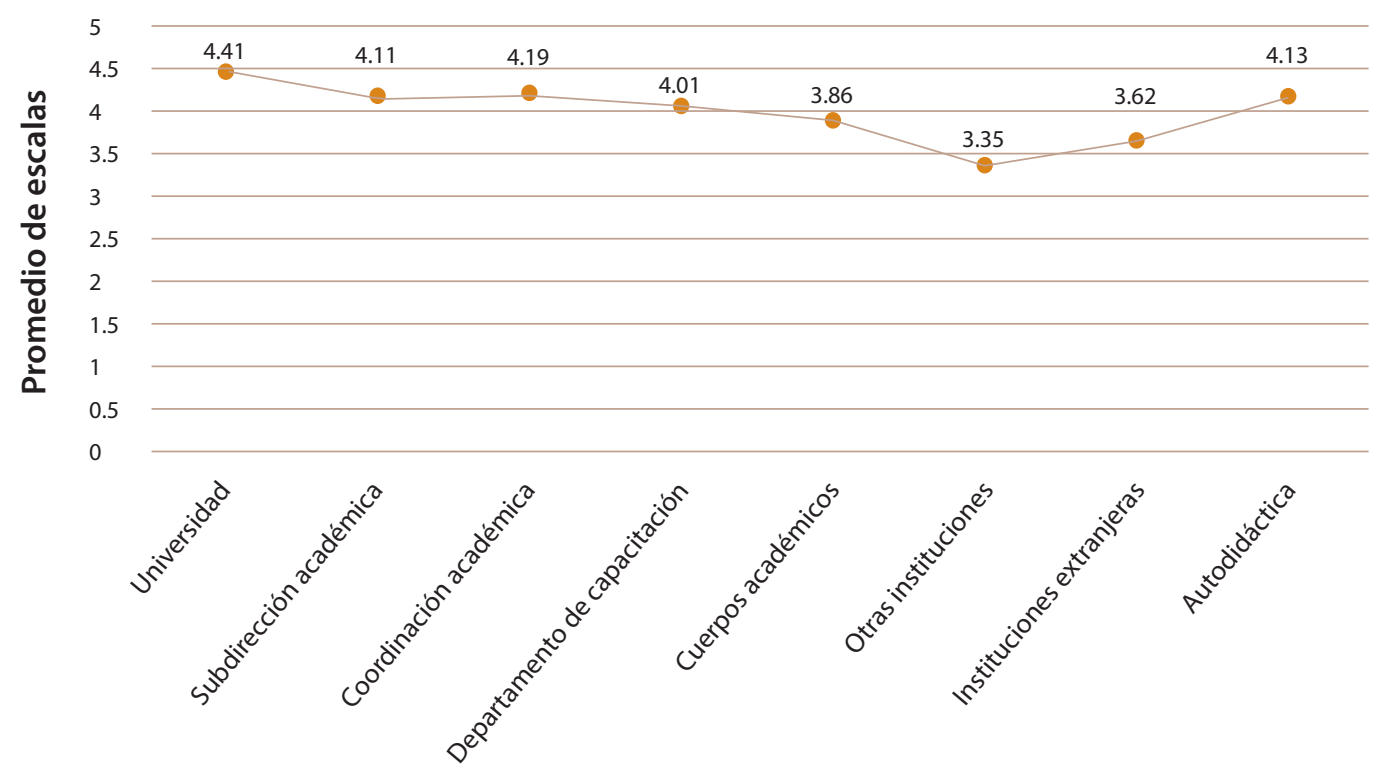

Instancias que aportan a la formación

Nota: Elaboración propia. Encuesta a profesorado de ingeniería, 2018.

Las preguntas abiertas que solicitaron propuestas para favorecer la formación del profesorado se agruparon en recomendaciones sobre tipos de actividades y contenidos demandados por el personal docente. 
http://doi.org/10.15359/ree.25-3.32

http://www.una.ac.cr/educare

educare@una.ac.cr

\section{Propuestas sobre tipos de actividades que prefieren, e intereses en temáticas que son necesarias para la preparación del personal docente}

Los tipos de actividades que recomendó el profesorado con mayor frecuencia en las respuestas fueron: cursos de actualización, visitas y/o pasantías por empresas e industrias, talleres de intercambio, y proyectos de investigación; en estos últimos se insistió mucho su impacto para el desarrollo científico del profesorado.

En segundo nivel, con menor frecuencia de las respuestas, se propusieron actividades metodológicas de cátedra y de coordinación de carrera para el dominio de contenidos disciplinares, las actividades de supervisión y monitoreo del proceso, y entrenamientos prácticos con tutoría de personas expertas.

En cuanto a las temáticas necesarias para la preparación del personal docente, los temas que solicitaron para dar respuesta a sus expectativas de superación aparecen en la Tabla 1. Aparecen por orden de elección; los más solicitados se corresponden con la tecnología educativa, el trabajo en equipo y el liderazgo. Se observó una menor atención a la formación didáctica y pedagógica, la gestión de proyectos y la investigación.

Tabla 1: Propuesta de temas por parte de docentes

\begin{tabular}{lc} 
Temas requeridos según docentes encuestados(as) & $\%$ de respuestas \\
\hline Tecnología educativa y uso de las TIC & $90 \%$ \\
Trabajo en equipo & $80 \%$ \\
Liderazgo & $80 \%$ \\
Vinculación industria-alumno(a) & $70 \%$ \\
Certificaciones internacionales & $60 \%$ \\
Software educativo en ciencias básicas & $50 \%$ \\
Demandas de las personas empleadoras y perfil de la persona egresada & $40 \%$ \\
Redacción científica & $30 \%$ \\
Didáctica y pedagogía & $20 \%$ \\
Gestión de proyectos & $10 \%$ \\
Metodología de investigación & $10 \%$ \\
\hline
\end{tabular}

Nota: Elaboración propia. Encuesta a profesorado de ingeniería, 2018. 
http://doi.org/10.15359/ree.25-3.32

\section{b) La práctica del personal docente en el proceso formativo del ingeniero o la ingeniera}

\section{Percepción del grado de protagonismo del estudiantado}

La independencia cognoscitiva del estudiantado tiene relación con el modo en que su docente guía el proceso. Con este propósito se ofrecieron variantes; un extremo representa la docencia en que el profesorado tiene el rol predominante y el estudiantado es poco independiente; el extremo opuesto representa al estudiantado con máximo protagonismo. El resultado general según el procesamiento de la escala de Likert indica la percepción del profesorado sobre el protagonismo del estudiantado con valor de 2.84, en escala máxima de 5.

El gráfico de distribución de respuestas por los cinco niveles de la escala muestra que el profesorado percibió insuficiencias en el protagonismo de sus estudiantes, si se observa que los niveles "muy bajo" y "poco" de protagonismo estudiantil en ingeniería suman $45.0 \%$. (Figura 2)

Este problema no radica solo en la acción del profesorado, y tiene alguna relación con los estilos de aprendizaje. Personas investigadoras se han referido a esto en el contexto mexicano (MoralesRamírez et al., 2012; Ortiz Ojeda y Canto Herrera, 2013); así, el primero encontró que el estudiantado se inclina más hacia los estilos pragmático y activo, sobre los estilos teórico y reflexivo. Significa que prefieren las actividades de aplicación sobre aquellas que requieren pensamiento analítico.

De aquí se deriva que es necesario para el personal docente lograr cambios en sus metodologías de enseñanza que produzcan transformación en los niveles de protagonismo, entendido como una actuación del estudiantado con mayor independencia y autogestión de su aprendizaje. De este modo, se configura como una necesidad la preparación docente para lograrlo, lo que resulta muy oportuno en la época actual, pues se avizora un incremento de la educación a distancia y reducción de la modalidad educativa presencial del alumnado en clases tradicionales.

Figura 2: Percepción del profesorado sobre el protagonismo del estudiantado. En porciento (\%)

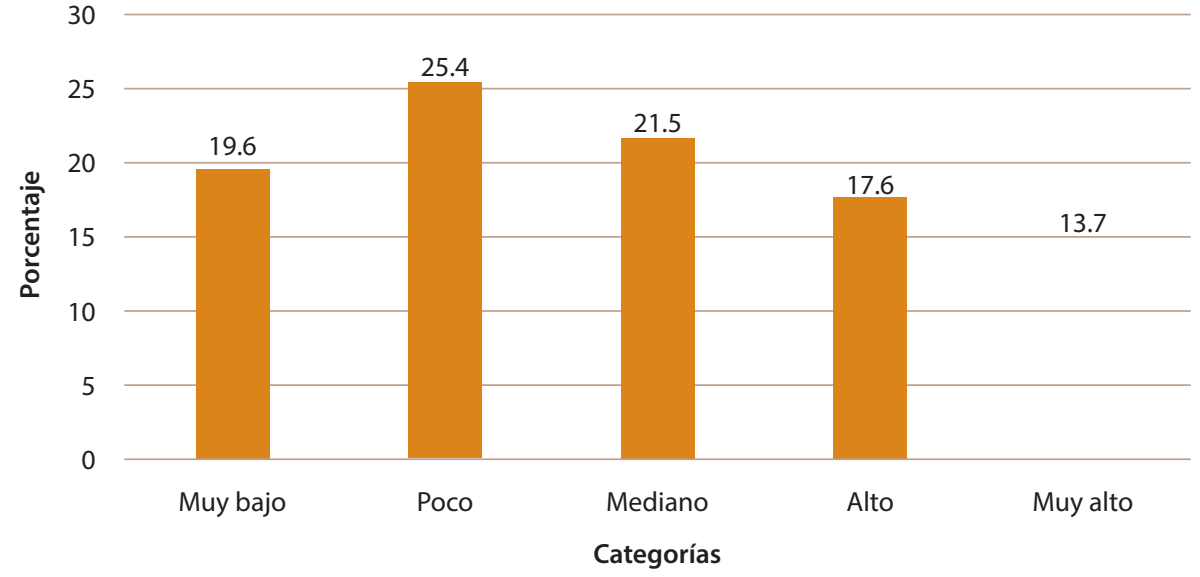

Nota: Elaboración propia. Encuesta a profesorado de ingeniería, 2018. 
http://doi.org/10.15359/ree.25-3.32

http://www.una.ac.cr/educare

educare@una.ac.cr

\section{Criterios sobre actividades docentes que están presentes en la formación de sus estudiantes}

Esta variable se analizó mediante la valoración de un listado predefinido de actividades que se consideraron adecuadas actualmente. El profesorado indicó para cada actividad si estaba presente en su docencia en tres niveles: alto, medio, y bajo. El resultado permitió conformar grupos según la calificación que asignaron a cada uno de los aspectos. La Tabla 2 muestra el agrupamiento que se conformó como tendencia general, en base a la frecuencia de las respuestas. Como se observa en la tabla, las actividades que los profesores y profesoras realizan con mayor frecuencia se corresponden con las que tradicionalmente se han relacionado con el aspecto académico. Sin embargo, en menor medida participan en la gestión institucional, elaboran y ejecutan proyectos de investigación y vinculan el proceso formativo con el entorno. La atención individual a su clase y la relación de lo académico con lo investigativo son las actividades que muestran un nivel más bajo de realización.

Tabla 2: Criterios sobre la ejecución de actividades del personal docente en la formación del estudiantado

\begin{tabular}{|c|c|}
\hline Nivel de ejecución & Actividades del listado \\
\hline Nivel alto & $\begin{array}{l}\text { - Organiza y motiva situaciones de aprendizaje. } \\
\text { - Gestiona la progresión de los aprendizajes en el estudiantado. } \\
\text { - Implica al estudiantado en investigaciones y otras tareas. } \\
\text { - Trabaja en equipo con colegas y con estudiantes. } \\
\text { - Organiza su propia formación continua como profesor(a). } \\
\text { - Usa las TIC para la enseñanza de la ingeniería. }\end{array}$ \\
\hline Nivel medio & $\begin{array}{l}\text { - Participa en la gestión institucional a diferentes niveles. } \\
\text { - Vincula el proceso formativo con el entorno cercano. } \\
\text { - Elabora y ejecuta proyectos de investigación e innovación. } \\
\text { - Implica al estudiantado en su aprendizaje, en investigaciones y otras tareas. } \\
\text { - Desarrolla competencias didácticas para enseñanza de la ingeniería. }\end{array}$ \\
\hline Nivel bajo & $\begin{array}{l}\text { - Aplica mecanismos de reconocimiento y estimulación al estudiantado. } \\
\text { - Prepara ponencias oartículosyen general socializa resultados de investigación }\end{array}$ \\
\hline
\end{tabular}

Nota: Elaboración propia. Encuesta a profesorado de ingeniería, 2018.

Los resultados indican la necesidad de priorizar la preparación en aquellas actividades que obtuvieron valoración menor. Entre ellas resultó insuficiente la actividad de estímulo y reconocimiento al estudiantado. Sin duda el profesorado debe preocuparse, y puede fortalecer 
http://doi.org/10.15359/ree.25-3.32

su trabajo educativo mejorando el componente afectivo del proceso. Desde el punto de vista axiológico se trataría del desarrollo de valores de empatía y compromiso. En esto coincide Álvarez (2011) cuando enuncia que son funciones importantes acompañar, orientar y guiar el trabajo y la búsqueda de cada estudiante; y promover el desarrollo integral y su mejoramiento continuo.

\section{Valoración sobre temas pertinentes y sobre prioridades a considerar por la persona docente en la formación en ingeniería}

En la investigación se propuso valorar un listado que se definió en base a fuentes consultadas y compatibilizado con los enunciados de los planes de estudio. El profesorado emitió sus criterios acerca del grado en que incluyen dichos contenidos en sus asignaturas. Los valores expresan el grado medio de introducción que el grupo manifiesta, calculado como promedio en una escala de 5 puntos (Tabla 3).

Los contenidos que alcanzan promedio en la zona baja son los temas del medio ambiente y la ingeniería, así como la cultura e identidad. Estos deben recibir prioridad para poder fortalecerlos mediante el proceso docente.

Tabla 3: Valoración del profesorado sobre contenidos que son pertinentes en el proceso docente actual. Promedio general del grupo

\section{Contenidos que la persona docente debe introducir Introducción del contenido. en sus materias: Promedio grupal (máximo: 5)}

Adelantos de la tecnología a nivel mundial

Desarrollo tecnológico sustentable del país

Valores y dilemas éticos de la profesión

Comunicación oral y escrita

Uso de idioma extranjero, uso de bibliografía en inglés

Uso de software profesional de ingeniería

Problemática social y económica relacionada con la ingeniería

Problemas del medio ambiente y la ingeniería

Cultura e identidad

2.87

Nota: Elaboración propia. Encuesta a profesorado de ingeniería, 2018. 
http://doi.org/10.15359/ree.25-3.32

http://www.una.ac.cr/educare

educare@una.ac.cr

Otra sección del cuestionario recogió información sobre prioridades que se deben tener en cuenta según el profesorado. Aquí no se utilizó un listado predefinido, sino lo que consideraron pertinente y útil en la actualidad. Como se aprecia en la gráfica, tuvieron mucha relevancia tres elementos: el trabajo en equipo, el emprendimiento y el pensamiento crítico (Figura 3).

Figura 3: Aspectos que se deben priorizar en las diferentes materias por el profesorado

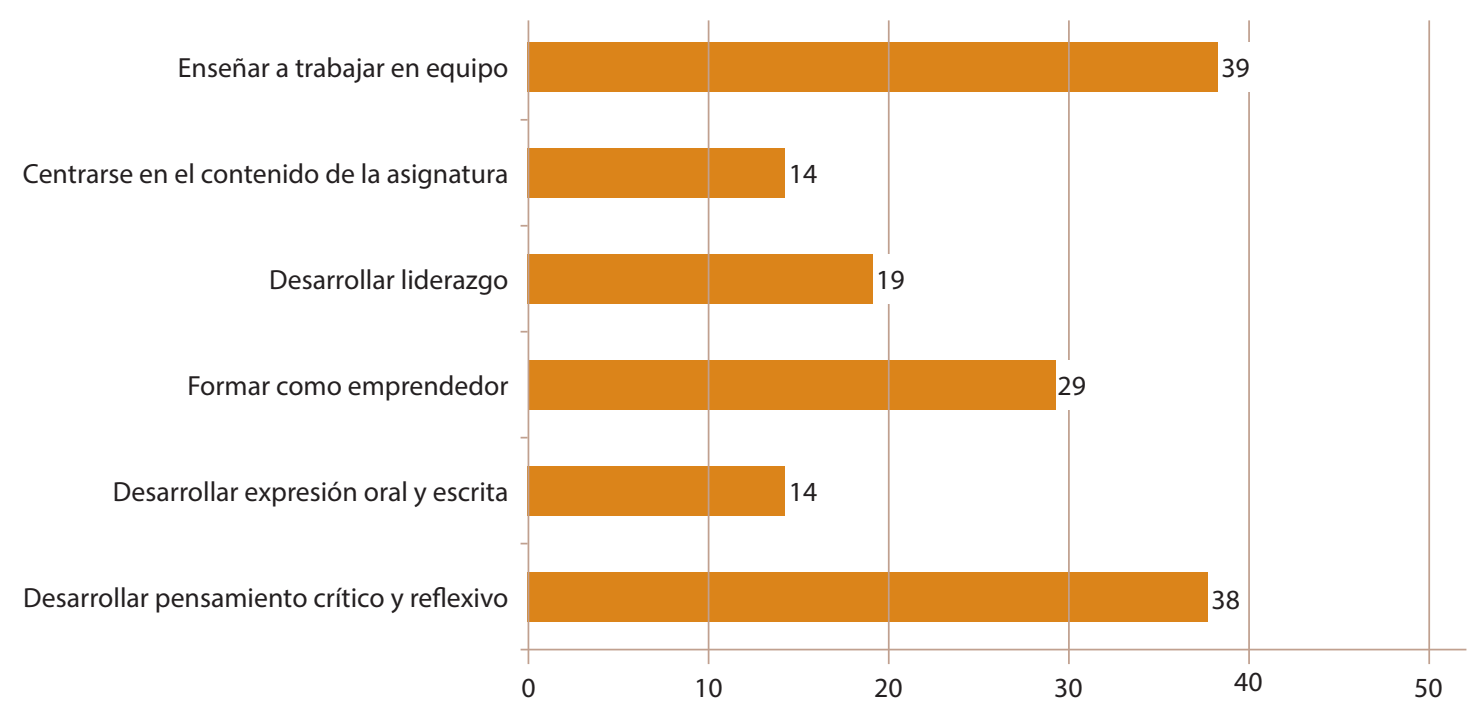

Nota: Elaboración propia. Encuesta a profesorado de ingeniería, 2018.

\section{Competencias transversales que se deben fortalecer en el proceso formativo estudiantil}

Para indagar sobre competencias deseadas en el estudiantado se propuso un listado al personal docente, y se realizó una selección múltiple; cada elemento fue ubicado en una categoría desde el menor al mayor desarrollo, según la apreciación del profesorado. El resultado grupal aportó varias competencias que fueron en la parte baja o de insuficiente desarrollo en el estudiantado. Los resultados pueden retroalimentar la labor del personal docente para influir en estas, en el sentido de prepararse teórica y metodológicamente para promoverlas en el proceso docente.

Las que valoraron como menos logradas y por consiguiente con mayores dificultades se relacionaron con: pensamiento estratégico, puntualidad en el cumplimiento de tareas, dominio de idioma extranjero, comunicación oral y escrita, gestión del tiempo para cumplir sus compromisos, autoaprendizaje y responsabilidad con su formación, interpretar datos e información, y pensamiento matemático. Es notorio que el desarrollo más deficiente se encuentra en las llamadas competencias blandas, que potencian la formación integral de los estudiantes. 
http://doi.org/10.15359/ree.25-3.32

\section{c) Ámbito personal del profesor y la profesora}

\section{Satisfacción personal de quienes ejercen la docencia respecto a su formación}

Para analizar sobre la satisfacción personal se utilizó una pregunta en escala de Likert. La satisfacción personal que expresaron los encuestados y encuestadas se manifestó en cinco niveles, relacionados con el proceso de formación por el cual han transitado. Las respuestas en las categorías "satisfecho(a)" y "totalmente satisfecho(a)" constituyeron una cantidad significativa, y sumaron el $68.0 \%$. Se puede asumir que el personal docente está satisfecho y que las actividades en que participan satisfacen necesidades y expectativas para su trabajo académico (Figura 4).

Figura 4: Satisfacción del personal docente con la formación mediante las actividades en que han participado. En porciento (\%)

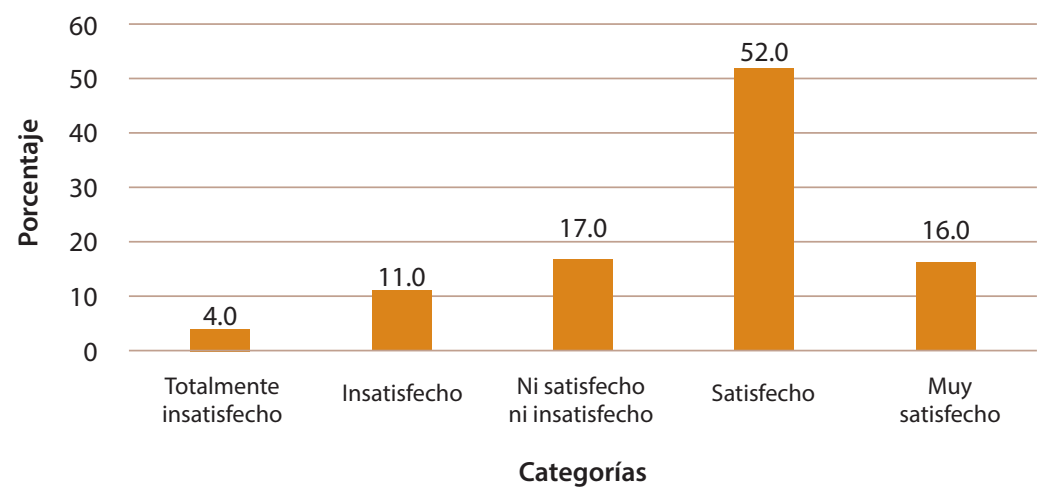

Nota: Elaboración propia. Encuesta a profesorado de ingeniería, 2018.

\section{Percepción de cambios por parte del personal docente}

En la percepción del profesorado sobre cambios originados por las actividades en que ha participado, el criterio que obtiene mejor valoración es el correspondiente a cambios en su persona (79.5\%); y el resultado con menor valor es la aplicación al proceso docente de los saberes adquiridos (73.5 \%). Esto último significa que en la superación del profesorado para su trabajo docente se pueden reforzar habilidades o competencias para la práctica, para implementar metodologías de enseñanza, innovar en el proceso, introducir contenidos y otras acciones. Aunque en general la transformación percibida no se valora negativamente si se observan las respuestas dadas a los cuatro criterios de transformación (Tabla 4). 
http://doi.org/10.15359/ree.25-3.32

http://www.una.ac.cr/educare

educare@una.ac.cr

Tabla 4: Percepción del personal docente sobre cambios originados por las actividades de superación que ha recibido

Percepción de cambios:

En su persona integralmente

En su calidad como docente

En los resultados de sus estudiantes

En la aplicación de lo aprendido a la docencia
Respuestas afirmativas del profesorado (\%)

$76.0 \%$

$74.3 \%$

$73.5 \%$

Nota: Elaboración propia. Encuesta a profesorado de ingeniería, 2018.

\section{Cualidades relacionadas con el perfil docente}

Esta pregunta tuvo dos partes: la primera fue una lista de selección y ordenamiento y la segunda consiste en un enunciado abierto para expresar una palabra que defina al profesorado de ingeniería.

(a) En la primera parte se propuso un listado predefinido de cualidades personales deseadas en el profesorado, para seleccionar tres y asignarles un orden. La selección del personal docente se expresó en tres de mayor preferencia: flexibilidad, actitud positiva, y dedicación. El resultado de la selección de cualidades se expone en el siguiente gráfico (Figura 5).

Figura 5: Preferencia sobre cualidades del profesorado, resultado de la selección en primer lugar. En porciento (\%)

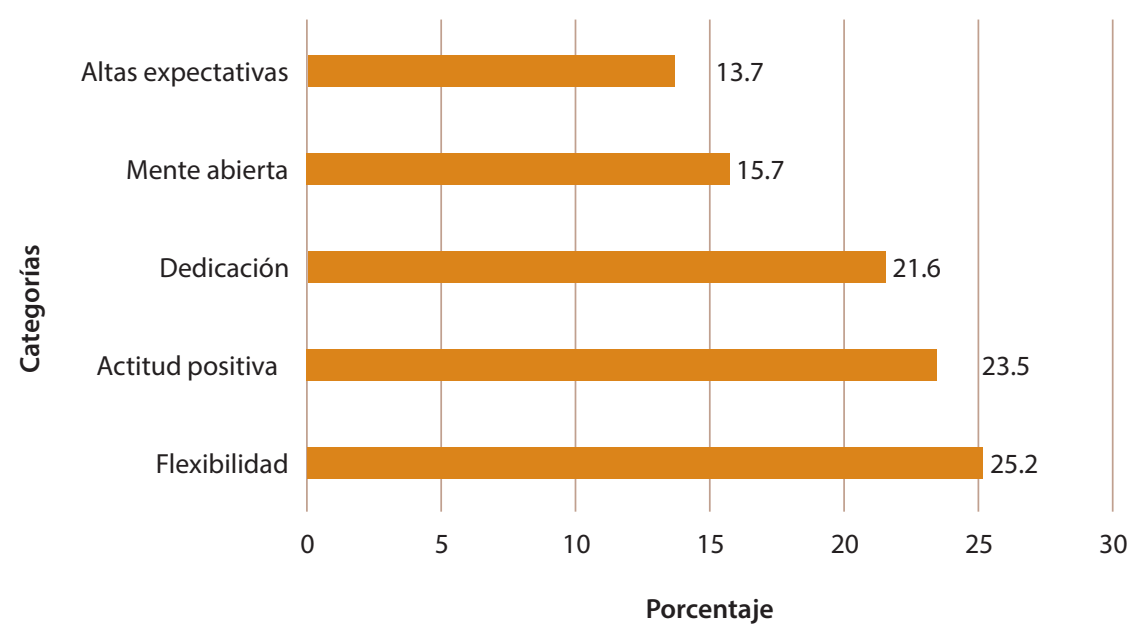

Nota: Elaboración propia. Encuesta a profesorado de ingeniería, 2018. 
http://doi.org/10.15359/ree.25-3.32

(b) Cualidades del profesorado. Mediante una pregunta de respuesta abierta se solicitó una cualidad deseada que define a un profesor o profesora de ingeniería, mediante una palabra. Los resultados se presentan gráficamente formando una nube de palabras, que es una técnica de visualización que consiste en la representación gráfica de las más recurrentes, de modo que los diferentes tamaños expresan la importancia de cada concepto que forma la nube (Peña Pascual, 2012). Para esta investigación, el tamaño y ubicación de las palabras se corresponden con la frecuencia en que fueron mencionadas las cualidades del profesorado ideal de ingeniería.

El sentido de estas respuestas se asume como el deber ser o la cualidad deseada, y todas de conjunto pueden ayudar a construir un enunciado de síntesis acerca del profesorado de ingeniería, en base a los conceptos aportados por las personas encuestadas.

En la respuesta del profesorado el concepto de innovador(a) resultó la cualidad predominante. Le sigue en lugar destacado la representación del profesorado como mentor y guía de sus estudiantes. La dedicación y el compromiso se visualizan en un tercer orden, y seguidamente el profesorado creativo, ingenioso, disciplinado, y responsable. Por último, se aportan conceptos que definen al profesorado como formador, líder, exitoso, apasionado, prudente, actualizado, investigador y analítico (Figura 6).

Figura 6: Nube de palabras con las cualidades deseadas para el profesorado de ingeniería

\section{lider actualizado \\ formador investigador analítico ingenioso disciplinado mentor innovador dedicado comprometido exitoso responsable creativo apasionado prudente}

Nota: Elaboración propia. Encuesta a profesorado de ingeniería, 2018.

Los resultados obtenidos muestran varias aristas de las percepciones del personal docente de ingeniería que participó en el estudio. A pesar de que se observó una posición crítica de sus carencias formativas, en gran medida persisten visiones tradicionales del rol docente al ignorar dimensiones importantes de su trabajo en esta esfera. 
http://doi.org/10.15359/ree.25-3.32

http://www.una.ac.cr/educare

educare@una.ac.cr

\section{Conclusiones}

De acuerdo con la revisión de fuentes actuales relacionadas con el tema, los resultados del estudio realizado aportan elementos coherentes con las tendencias para la formación integral de quien egresa de carreras de ingeniería.

En la formación del profesorado de ingeniería intervienen diversos factores que se agruparon en tres ámbitos para realizar esta investigación: el institucional, desde el cual se planifican y ejecutan actividades de formación del personal docente; el profesional, relacionado con la ejecución del proceso formativo en el cual el profesorado realiza sus funciones; y el ámbito personal del profesorado: sus cualidades, preferencias, satisfacción.

Las percepciones sobre cualidades ideales del profesorado, las propuestas de temas a incluir, y los criterios de valoración sobre el contenido de actividades en la ejecución de la docencia ratificaron la necesidad de considerar a la innovación, el liderazgo, la creatividad y la actividad investigativa como aspectos relevantes para la preparación del personal docente de modo tal que trasciendan a la formación del estudiantado. Se observa que no siempre las personas participantes en el estudio son conscientes de sus necesidades formativas como docente. Sin embargo, diferentes investigaciones como la realizada por Zuluaga-Rendón et al. (2017) muestra que el estudiantado señala con precisión las carencias de sus profesores y profesoras en cuanto a la evaluación, el dominio del tema, la experiencia docente, la aplicación de temas a problemas reales de la ingeniería y otros aspectos psicosociales, tales como la motivación y la asertividad.

Los resultados del ámbito institucional indican que las relaciones interinstitucionales y la movilidad académica son posibilidades relevantes para favorecer la formación del personal docente, pero estos fueron los elementos que obtuvieron el menor promedio entre los que se evaluaron. En este caso, se deben aprovechar las potencialidades de estas vías por su valioso aporte al incremento del nivel de calidad del profesorado. Esta falta de proyección concuerda con la existencia de problemas no resueltos en cuanto a la transformación necesaria en el intercambio y la movilidad de estudiantes, profesores y profesoras de ingeniería para desarrollar una formación más universal, y en esto juega un papel fundamental el personal docente. Diversos trabajos muestran la necesidad de establecer un sistema de acreditación que facilite el reconocimiento internacional de los programas de ingeniería y quienes egresan de ellos (Metaute Paniagua et al., 2018).

En el ámbito profesional el profesorado señaló la necesidad de reforzar el trabajo formativo: el $42.8 \%$ de las personas encuestadas consideró como insuficiente la labor del profesorado para estimular y reconocer al estudiantado. Sobre los contenidos que introducen en sus clases se infiere la necesidad de fortalecer las temáticas sobre cultura e identidad, así como la relación de la ingeniería y medio ambiente. Además, según criterios del $76.4 \%$ del profesorado, se requiere 
http://doi.org/10.15359/ree.25-3.32

capacitación para el trabajo en equipo. Hoy día una formación docente en ingeniería que trascienda los aspectos técnicos está en en el centro de las investigaciones sobre este tema, ya que como apuntan Correia Barreiro y Bozutti (2017), los profesores y profesoras han de preparar a sus estudiantes a tomar decisiones, a colaborar en proyectos colectivos y otros aspectos que permiten realizar el trabajo con mayor efectividad, y esto se logra, entre cosas, a través de una adecuada comunicación docente-estudiante.

En el ámbito personal, las percepciones sobre el profesorado de ingeniería se expresaron en cualidades de tipo técnico-profesional (innovador(a), ingenioso(a)), educativo (formador(a), líder, mentor(a)), intelectual (analítico(a), investigador(a)), y ético (dedicado(a), responsable, prudente, disciplinado(a), comprometido(a)). Según criterio de las personas encuestadas, la cualidad más relevante fue la de innovación. Aunque efectivamente estas cualidades son indispensables para formar al ingeniero y la ingeniera que la sociedad requiere, una formación más integral precisa, como expresan Capote León et al. (2016), el fomento de la capacidad de comunicación, saber utilizar la información, el dominio de lenguas extranjeras y una adecuada instrucción económica y educación

Aunque los resultados muestran satisfacción del personal docente con la formación que ha alcanzado, desde las perspectivas de desarrollo de la ingeniería y lo que ello implica para el proceso formativo, los resultados permitieron identificar necesidades y propuestas de temas para la formación de este profesorado, a saber: elaboración y gestión de proyectos de investigación e innovación, metodologías de enseñanza que propicien el protagonismo y la independencia del alumnado, aplicación de tecnologías informáticas en el proceso formativo, y vinculación de estudiantado y profesorado de ingeniería con la industria. Se observa que las personas participantes, al menos en sus respuestas, denotan cierto desconocimiento de aspectos esenciales que, como formadores y formadoras, deben potenciar.

Los resultados obtenidos aportaron una base para continuar la investigación con alcance transformador, mediante propuestas específicas que propicien la formación de docentes de ingeniería de acuerdo con sus necesidades personales, profesionales y que se correspondan con las exigencias de la sociedad actual.

Los resultados obtenidos en este estudio deben analizarse tomando en cuenta las limitaciones posibles del mismo. En este sentido, aunque participaron profesores y profesoras de nueve carreras de ingeniería, una muestra de docentes en otros contextos pudiera aportar mayores elementos para el mejoramiento de la formación docente en ingeniería acorde a las necesidades y exigencias actuales.

\section{Declaración de Material complementario}

Este artículo tiene disponible, como material complementario:

-La versión preprint del artículo en https://doi.org/10.5281/zenodo.4796522 
http://doi.org/10.15359/ree.25-3.32

http://www.una.ac.cr/educare

educare@una.ac.cr

\section{Referencias}

Albor Calderón, M. G., Groce Ortiz, A. T. y Luna Ramírez, F. E. (2018). Análisis de la formación académica de ingenieros químicos industriales, como líderes en el ámbito laboral. Revista Electrónica Anfei Digital, 4(8), 1-10. https://www.anfei.mx/revista/index.php/revista/ article/view/414/1061

Álvarez, M. M. (2011). Perfil del docente en el enfoque basado en competencias. Revista Electrónica Educare, 15(1), 99-107. https://doi.org/10.15359/ree.15-1.7

Banda, F. (2018). Formación del profesor de ingeniería. Desafíos del tercer milenio. Cengage Learning Editores.

Beddoes, K. y Panther, G. (2018). Gender and teamwork: An analysis of professors' perspectives and practices. European Journal of Engineering Education 43(3), 330-343. https://doi.org/1 $\underline{0.1080 / 03043797.2017 .1367759}$

Cáceres Mesa, M., Lara Díaz, L., Iglesias León, C.M., García Cruz, R., Bravo López, G., Cañedo Iglesias, C. y Valdés Chaviano, O. (2003). La formación pedagógica de los profesores universitarios. Una propuesta en el proceso de profesionalización del docente. Revista lberoamericana de Educación 33(1), 1-15. https://doi.org/10.35362/rie3312900

Capote León, G. E., Rizo Rabelo, N. y Bravo López, G. (2016). La formación de ingenieros en la actualidad. Una explicación necesaria. Universidad y Sociedad, 8(1), 21-28. https://rus.ucf. edu.cu/index.php/rus/article/view/300/297

Castillo-Elizondo, J. A., Álvarez-Aguilar, N. T. y Treviño-Cubero, A. (2019). La vinculación como potenciadora de la formación profesional de profesionales de ingeniería: Propuesta de acciones con base en experiencias en la Universidad Autónoma de Nuevo León. Revista Electrónica Educare 23(2), 1-21. https://doi.org/10.15359/ree.23-2.11

Cederbaum, S. (2018). Aportes para el ejercicio de la docencia en ingeniería. Tecnología y Sociedad, (7), 49-62. https://erevistas.uca.edu.ar/index.php/TYS/article/view/1511/1432

Centro de Investigación para el Desarrollo. (2014). Encuesta de competencias profesionales 2014. ¿Qué buscan -y no encuentran- las empresas en los profesionistas jóvenes? Autor. http:// cidac.org/esp/uploads/1/encuesta_competencias_profesionales_270214.pdf

Cid Reborido, A. (2009). Formación del profesorado de ingeniería desde la teoría de la complejidad: Un estudio cualitativo [Tesis doctoral]. Universitat de Barcelona. https://www.tdx.cat/hand le/10803/1362;jsessionid=F6F5579DB2E188C0E25F74877ED1243C\#page $=4$

Correia Barreiro, S. y Bozutti, D. F. (2017). Desafíos y dificultades en la enseñanza de la ingeniería a la generación Z: Un caso de estudio. Propósitos y Representaciones, 5(2), 127-183. https:// dx.doi.org/10.20511/pyr2017.v5n2.163 
de Castro, M. (2011). La formación profesoral en la UTB. Ediciones Tecnológica de Bolívar. https:// www.utb.edu.co/sites/web.unitecnologica.edu.co/files/descargas/2-formacion 0.pdf

Denzin, N. K. y Lincoln, Y. S. (Coords.). (2012). Manual de investigación cualitativa (Vol. 1: El campo de la investigación cualitativa). Gedisa. http://www.ceil-conicet.gov.ar/wp-content/ uploads/2015/10/Denzin-Intro-Handbok.rtf

Duque, M., Celis, J. y Camacho, A. (2011). Cómo lograr alta calidad en la educación de los ingenieros: Una visión sistémica. Revista Educación en Ingeniería, 6(12), 48-60. https://doi. org/10.26507/rei.v6n12.122

Flores Aguilar, M. D., Franco Ortega, J. C., Raygoza Solís, L. y Vargas Cortez, C. del R. (2018). La argumentación, fundamento en la formación de líderes de ingeniería. Revista Electrónica ANFEI Digital, 4(8), 1-10 https://www.anfei.mx/revista/index.php/revista/issue/view/13/showToc

Fondón, I., Madero, M. J. y Sarmiento, A. (2010). Principales problemas de los profesores principiantes en la enseñanza universitaria. Formación Universitaria, 3(2), 21-28. https:// doi.org/10.4067/S0718-50062010000200004

García Salinero, J. (2004). Estudios descriptivos. Nure Investigación, (7), 1-3. https://www. nureinvestigacion.es/OJS/index.php/nure/article/view/180/163

López Serrano, M. E. (2019). El desarrollo profesional de docentes. Una propuesta desde la complejidad. RIDE. Revista Iberoamericana para la Investigación y el Desarrollo Educativo, 10(19), 1-17. https://doi.org/10.23913/ride.v10i19.492

Mas Torelló, Ó. (2011). El profesor universitario: Sus competencias y formación. Profesorado. Revista de currículum y formación del profesorado, 15(3), 195-211. https://dialnet.unirioja. es/servlet/articulo?codigo $=4091117$

Metaute Paniagua, P. M., Flórez Osorio, G. A., Rúgeles Contreras, P. A. y Castaño, D. A. (2018). La dinamización de las estrategias pedagógicas actuales: Una necesidad aplicable a los procesos de enseñanza y aprendizaje de los estudiantes de ingeniería del siglo XXI. Revista Lasallista de Investigación, 15(1). 46-56. https://doi.10.22507/rli. v15n1a4

Morales Ramírez, A., Alviter Rojas, L. E., Hidalgo Cortés, C., García Lozano, R. Z. y Molinar Solís, J. E. (2012). Estilos de aprendizaje en estudiantes universitarios de ingeniería en computación e informática administrativa. Revista Estilos de Aprendizaje, 5(9), 156-168. http://revistaestilosdeaprendizaje.com/article/view/953

Ortiz Ojeda, A. F. y Canto Herrera, P. J. (2013). Estilos de aprendizaje y rendimiento académico en estudiantes de ingeniería en México. Revista Estilos de Aprendizaje, 6(11), 160-177. http:// revistaestilosdeaprendizaje.com/article/view/978 
http://doi.org/10.15359/ree.25-3.32

http://www.una.ac.cr/educare

educare@una.ac.cr

Peña Pascual, I. (2012). Posibilidades de las "nubes de palabras" (word clouds) para la elaboración de actividades de contenido cultural en el aula de AICLE. En R. Breeze, F. Jiménez Berrio, C. Llamas Saíz, C. Martínez Pasamar y C. Tabernero Sala (eds.), Teaching Approaches to CLIL. Propuestas docentes en AICLE, (pp. 249-264). Servicio de Publicaciones Universidad de Navarra. https://www.researchgate.net/publication/259484477 Teaching approaches to CLIL Propuestas docentes en AICLE

Pusca D. y Northwood, D. O. (2018). Curiosity, creativity and engineering education. Global Journal of Engineering Education, 20(3), 152-158 http://www.wiete.com.au/journals/GJEE/ Publish/vol20no3/01-Pusca-D.pdf

Salinas Cruz, A. L. (2019). Las competencias tutoriales practicadas por el docente tutor del CECyT 16 Hidalgo: Desde la perspectiva de los lineamientos establecidos para esta función por la ANUIES [Tesis de maestría]. Instituto Politécnico Nacional.

Teerijoki, H. y Murdock, K. A. (2014). Assessing the role of the teacher in introducing entrepreneurial education in engineering and science courses. The International Journal of Management Education, 12(3), 479-489. https://doi.org/10.1016/j.jjme.2014.05.005

Vázquez Toledo, S., Liesa Orús, M. y Bernal Agudo, J. L. (2015). Análisis de la formación en liderazgo en las organizaciones educativas: Un estudio en la Comunidad Autónoma de Aragón. Revista Electrónica Interuniversitaria de Formación del Profesorado, 18(3), 39-54. https://doi.org/10.6018/reifop.18.3.194881

Vega González, L. R. (2013). La educación en ingeniería en el contexto global: Propuesta para la formación de ingenieros en el primer cuarto del siglo XXI. Ingeniería Investigación y Tecnología, 14(2), 177-190. https://doi.org/10.1016/S1405-7743(13)72235-2

Zaldívar Acosta, M., Canto Herrera, P. J. y Rubio Quintero Mármol, N. L. (2018). La calidad de los posgrados de formación docente en México, 48(1), 131-142. https://doi.org/10.30827/ publicaciones.v48i1.7333

Zuluaga Rendón, Z., Corredor, J. A., Quintero, J. M., Ramírez Echeverry, J. J. y Olarte, F. A. (2017) ¿Qué es una buena clase en ingeniería desde el punto de vista de los estudiantes? Revista Educación en Ingeniería, 12(23), 83-92. https://doi.org/10.26507/rei.v12n23.740 